\title{
Miser sur les capacités citoyennes pour faire face à la crise climatique
}

Audrey Groleau, Isabelle Arseneau et Chantal Pouliot

\section{OpenEdition}

Édition électronique

URL : https://journals.openedition.org/ere/7600

ISSN : 2561-2271

\section{Éditeur}

Centr'ERE

\section{Référence électronique}

Audrey Groleau, Isabelle Arseneau et Chantal Pouliot, « Miser sur les capacités citoyennes pour faire face à la crise climatique ", Éducation relative à l'environnement [En ligne], Volume 17-1 | 2022, mis en ligne le 27 janvier 2022, consulté le 03 février 2022. URL : http://journals.openedition.org/ere/7600

Ce document a été généré automatiquement le 3 février 2022.

La revue Éducation relative à l'environnement est mise à disposition selon les termes de la Licence Creative Commons Attribution - Pas d'Utilisation Commerciale 4.0 International. 


\title{
Miser sur les capacités citoyennes pour faire face à la crise climatique
}

\author{
Audrey Groleau, Isabelle Arseneau et Chantal Pouliot
}

1 La gestion sociopolitique de la crise climatique actuelle exige de repenser les rôles et capacités des scientifiques et des citoyen.ne.s. Depuis quelques années, on constate, d'une part, que des scientifiques sortent de leur laboratoire et prennent la parole pour alerter les décideurs quant aux risques de l'inaction politique (Ripple et coll., 2019). D'autre part, on remarque qu'un grand nombre de citoyen.ne.s font la démonstration de leur capacité à saisir des enjeux complexes et à mettre de l'avant des savoirs spécifiques et des actions efficaces (Akrich, Barthe et Rémy, 2010). L'éducation aux sciences poursuit d'ailleurs la visée - à la fois transformatrice et critique de l'enchevêtrement des sciences dans la société - de former de tels citoyen.ne.s (Sjöström et Eilks, 2018). Cette vision de l'alphabétisation technoscientifique passe entre autres par l'enseignement des questions socialement vives et s'intègre dans l'approche du bien-être pour les individus, les sociétés et les environnements (en anglais, WISE) (Bencze, 2017). Cet enseignement et cette approche ont en commun de faire une large place aux réflexions, discussions et actions et de bien se prêter à des thèmes à teneur environnementale comme la crise climatique. C'est à la lumière de ces enjeux contemporains et de ces visées démocratiques que nous adoptons, dans nos travaux de recherche et dans nos cours, une posture dite antidéficitaire face aux citoyen.ne.s.

\section{Le concept de déficit citoyen et la posture antidéficitaire}

2 Il n'est pas rare de lire dans les médias que les citoyen.ne.s ne maitrisent pas les savoirs scientifiques de base relatifs aux gaz à effet de serre ou aux combustibles fossiles, qu'ils.elles ne sont pas en mesure de comprendre les enjeux scientifiques et technologiques liés aux changements climatiques, ni de prendre des décisions individuelles et collectives rationnelles en ce qui concerne leurs déplacements, la gestion de leurs déchets et l'achat de biens et services. Le concept de déficit citoyen 
correspond à cette idée selon laquelle les citoyen.ne.s souffriraient de déficits de connaissances, de compréhension et d'intérêt face aux technosciences (Callon, 1998 ; Pouliot, 2009). Aujourd'hui, avec l'exposition aux fausses nouvelles, le déficit citoyen s'étend à leur supposée incapacité de distinguer le vrai du faux (Groleau, 2019).

Or, depuis près de 30 ans, le concept de déficit citoyen est examiné et critiqué dans le domaine des science studies. En réponse à la question de la confiance envers les sciences et les institutions scientifiques débattue depuis les années 90, des auteur.e.s, dont Irwin (1995) et Wynne (1992), ont formulé de vigoureuses critiques à l'égard de la communication unidirectionnelle des scientifiques vers les " profanes » et du caractère exceptionnaliste accordé à l'expertise. Wynne (1991) défendait alors l'idée selon laquelle les recherches issues du champ du public understanding of science gagneraient à choisir des approches méthodologiques permettant de comprendre les points de vue et les connaissances citoyens de façon plus détaillée et nuancée. En effet, les recherches d'alors en arrivaient souvent à la conclusion que les citoyen.ne.s, à la suite de la complétion de questionnaires ne leur offrant pas la possibilité d'approfondir leurs réponses, étaient ignorant.e.s. Ces auteur.e.s appelaient à considérer le caractère légitime et plus large des préoccupations citoyennes (Irwin, 2014). Différentes études, notamment celles réalisées auprès d'éleveurs.euses de moutons (Wynne, 1992) et de personnes vivant avec le VIH (Epstein, 1995), ont mis en lumière que les savoirs citoyens sont utiles - voire essentiels - pour s'approprier les questions technoscientifiques. Plus encore, ces études ont contribué à montrer que les citoyen.ne.s peuvent développer une crédibilité, une expertise citoyenne et, dans plusieurs cas, une expertise scientifique.

4 En réponse à ces préoccupations, Callon (1998) a proposé trois modèles pour interpréter les interactions entre les citoyen.ne.s et les scientifiques. Selon le premier modèle, celui de l'instruction publique (aussi appelé modèle du déficit), les citoyen.ne.s seraient déficitaires de connaissances et, par conséquent, irrationnels lors de la prise de position et de décisions. Pour ces raisons, ils.elles s'avèreraient incapables de participer aux débats et à la production des savoirs scientifiques. Dans ce contexte, un des rôles des scientifiques est d'informer les citoyen.ne.s afin de combler leurs déficits. Dans le deuxième modèle, celui du débat public, on considère que les savoirs des scientifiques et des citoyen.ne.s sont complémentaires. En effet, ce modèle considère que les savoirs non scientifiques des citoyen.ne.s peuvent enrichir le cadrage de la recherche et favoriser un usage pertinent des savoirs scientifiques (Pouliot et Godbout, 2014). Toutefois, si les citoyen.ne.s peuvent contribuer aux débats, ils ne peuvent toujours pas prendre part à la production des savoirs scientifiques. Dans le troisième modèle, celui de la coproduction des savoirs, les citoyen.ne.s sont en mesure de contribuer de façon pertinente à la production des savoirs scientifiques. Leurs connaissances locales et contextualisées, difficiles à percevoir pour une personne œuvrant hors de leur milieu, et leurs observations liées à l'évolution d'une situation (par exemple, une maladie avec laquelle ils.elles vivent ou la pollution d'un cours d'eau) sont nécessaires pour documenter les situations et agir sur elles. Callon, Lascoumes et Barthe (2001), dans leur ouvrage Agir dans un monde incertain, de même qu'Akrich, Barthe et Remy (2010), dans Sur la piste environnementale - Menaces sanitaires et mobilisations profanes, recensent un large éventail de moments et de stratégies permettant aux citoyen.ne.s concerné.e.s de contribuer aux débats, aux prises de 
décisions et à la production de savoirs lorsque des questions technoscientifiques émergent.

5 En somme, ces travaux ont montré qu'il est inexact de prétendre que les citoyen.ne.s sont ignorant.e.s, irrationnel.le.s ou désintéressé.e.s relativement aux technosciences, en plus de mettre en lumière qu'il existe des manières d'envisager les relations entre les citoyen.ne.s et les scientifiques s'éloignant de la posture déficitaire et que les citoyen.ne.s peuvent contribuer de façon pertinente aux débats et aux prises de décisions.

6 Sur la base de ces constats, comment expliquer que le concept de déficit citoyen demeure d'actualité, bien qu'il ait vivement été critiqué et que, manifestement, il s'agit d'un concept bancal ? À nos yeux, cela s'explique par le fait qu'il permet non seulement de reconduire le statu quo, mais qu'il peut aussi être utile, pour certaines personnes en position d'autorité, tel que l'observe Jasanoff (2008), de tenter de miner l'expertise qu'on attribue à différents groupes sociaux, notamment les citoyen.ne.s. En effet, et comme le fait remarquer Wynne (2006), l'idée de déficit a souvent été employée pour expliquer l'opposition des citoyen.ne.s à des projets, en laissant entendre que leur opposition s'explique par leur incompréhension des avantages de ceux-ci. Dans le même ordre d'idées, on peut évoquer l'argument qu'ils.elles rejettent les projets pour ne pas nuire à leur propre qualité de vie (une stratégie relevée par Batellier et Maillé, 2017). Ainsi, en gardant vivante l'idée de déficit citoyen, les relations de pouvoir asymétriques entre les citoyen.ne.s et les autorités scientifiques et politiques peuvent être maintenues. Les décideurs.euses peuvent alors se sentir légitimes de prendre des décisions unilatérales, en ne se préoccupant pas d'intégrer les savoirs, les préoccupations et les points de vue citoyens. Pour ces raisons, nous nous opposons vigoureusement, dans nos travaux de recherche et dans notre enseignement, à l'adoption d'une posture déficitaire envers les citoyen.ne.s. Autrement dit, nous entretenons une posture antidéficitaire (Groleau, 2017) ${ }^{1}$.

\section{« Lire en positif »}

7 Cette posture s'actualise notamment par la lecture «en positif " (Charlot, 1997) que nous faisons des situations que nous documentons. Cela signifie que nous nous intéressons à ce que nos étudiant.e.s et les participant.e.s à nos recherches savent, font et sont (plutôt qu'à ce qu'ils ne savent, ne font et ne sont pas). Notre posture antidéficitaire se traduit également dans le choix de nos projets de recherche: documenter les manières dont des citoyen.ne.s s'approprient des questions technoscientifiques d'actualité et agissent sur elles (par exemple, Pouliot, 2015); documenter les rapports à l'expertise scientifique d'étudiant.e.s qui seront amené.e.s à enseigner les questions technoscientifiques ou à agir à titre d'expert.e.s (par exemple, Groleau, 2017); documenter les points de vue de scientifiques qui agissent dans le contexte de questions environnementales ou sanitaires sur leurs propres actions et sur les rôles et les capacités qu'ils.elles s'attribuent et attribuent aux citoyen.ne.s (par exemple, Arseneau, 2019). Cette lecture en positif est enfin visible dans le choix des approches que nous privilégions dans notre enseignement et qui nous permettent de mettre en lumière les capacités citoyennes. 


\section{Pourquoi s'intéresser aux capacités citoyennes?}

8 Cette notion de capacité revêt un intérêt particulier puisqu'elle fait contrepoids à celle de déficit, associée à une lecture en négatif des objets que nous analysons et dont nous discutons. Si la notion de capacité citoyenne n'a pas fait l'objet d'une conceptualisation formelle dans les recherches en science studies et en didactique des sciences (du moins à notre connaissance), elle nous apparait néanmoins féconde. Nous associons la notion de capacité citoyenne à ce que les citoyen.ne.s sont en mesure de faire et de comprendre dans le contexte d'une question technoscientifique pour laquelle ils.elles considèrent être concerné.e.s. Ces capacités sont de divers ordres : elles peuvent être relatives à l'information (la comprendre ou l'évaluer par exemple), à l'apprentissage, à l'interaction, à la prise de décision, à l'action, etc.

9 Cette notion de capacité citoyenne nous apparaît particulièrement utile dans l'enseignement. En effet, pour que les apprenant.e.s s'engagent dans les apprentissages, dans la construction d'opinons, dans la production des savoirs de même que dans les débats et les processus de prises de décisions, il faut d'abord qu'ils.elles considèrent en avoir la capacité. Ainsi, en développant les capacités citoyennes, il devient possible de générer l'action sociopolitique, que ce soit en classe (par exemple par la mise en place d'une campagne de sensibilisation, par la rédaction d'une lettre à un.e élu.e) ou dans le contexte d'initiatives individuelles ou collectives réalisées dans la communauté.

\section{Comment s'y prendre?}

10 Une manière pertinente de s'intéresser aux capacités citoyennes en classe consiste à enseigner des questions technoscientifiques socialement vives, en particulier celles qui concernent l'environnement et la santé. Par définition, il s'agit de questions complexes, liées à plusieurs disciplines, qui sont à la fois vives dans les savoirs savants, dans la société et à l'école (Legardez et Simonneaux, 2006). Elles intéressent divers groupes d'acteurs.trices sociaux souhaitant intervenir dans les débats et les prises de décisions (Callon et coll., 2001). Pour ce faire, nous faisons appel à différentes stratégies pédagogiques que nous jugeons particulièrement utiles: l'ilot de rationalité interdisciplinaire (Maingain et Dufour, 2002), le jeu de rôles (Albe, 2005), le jeu de société (Groleau et Pouliot, 2014), l'enquête citoyenne (Pouliot, 2019), les recherches qui mènent à une action sociale (Bencze et Carter, 2011), pour n'en nommer que quelques-unes. Si chacune poursuit des visées différentes et adopte des modalités de réalisation spécifiques, elles ont en commun de mettre en lumière des capacités citoyennes. Si nous croyons que chacune de ces approches gagne à être explorée, nous avons choisi d'illustrer notre propos en examinant le potentiel pédagogique de deux productions médiatiques qui présentent une démarche d'enquête citoyenne, J'aime Hydro (Beaulieu, 2019) et Bras de fer (Seaborn et Seaborn, 2017), respectivement une pièce de théâtre et un film documentaires.

11 Dans la pièce J'aime Hydro dont elle est l'auteure, la comédienne Christine Beaulieu relate les étapes de l'enquête qu'elle a réalisée et qui l'a menée à se pencher sur la question suivante: pourquoi la société d'État Hydro-Québec continue-t-elle de construire des barrages hydroélectriques si elle dispose de surplus d'électricité qu'elle revend à perte ? Tout au long de son enquête, Christine Beaulieu s'approprie différents concepts technoscientifiques, pose des questions lors d'une commission sur l'énergie, 
lit des rapports, visite deux centrales hydroélectriques et rencontre des citoyen.ne.s, des expert.e.s, des syndicalistes, le président directeur-général d'Hydro-Québec et même le premier ministre du Québec. De leur côté, les documentaristes Jean-Laurence et Jonathan Seaborn, dans leur film Bras de fer, suivent la démarche de Véronique Lalande et Louis Duchesne, deux citoyen.ne.s du quartier Limoilou (à Québec), qui ont entamé une double démarche: documenter scientifiquement la problématique de l'empoussièrement anormal de leur quartier et mobiliser les citoyen.ne.s concerné.e.s. Afin de déterminer la composition et la provenance des poussières, Lalande et Duchesne feront analyser des échantillons en laboratoire, scruteront les caractéristiques géophysiques de la région, décortiqueront les recherches menées sur les poussières en milieu urbain, acquerront le vocabulaire technique, documenteront les épisodes de dispersion et produiront des rapports. Il et elle tiendront parallèlement des assemblées citoyennes, se présenteront au conseil de ville, parviendront à recadrer la dispersion de poussières comme un problème récurrent plutôt qu'un épisode unique, feront modifier les normes relatives à la qualité de l'air et déposeront deux actions collectives contre le Port de Québec et la compagnie Arrimage du Saint-Laurent, à qui il et elle attribuent les nuisances subies.

Si différentes manières d'exploiter ces œuvres en classe ont déjà été discutées (Arseneau et Groleau, 2020 ; Pouliot, 2019), nous sommes d'avis qu'un inventaire des capacités dont leurs protagonistes font preuve - notamment comprendre les tenants et aboutissants d'une problématique complexe, maitriser des savoirs technoscientifiques, en produire de nouveaux, se construire une opinion approfondie et nuancée, débattre et informer d'autres groupes d'acteurs.trices sociaux - serait une activité intéressante à réaliser en classe. En effet, on pourrait ensuite discuter des capacités citoyennes pertinentes à l'action relative à la crise climatique : comprendre l'effet de serre, être en mesure de bien expliquer entre autres quelles seront les conséquences des changements climatiques sur les communautés de l'Arctique, avoir réfléchi aux arguments soutenant le développement de la filière de l'hydrogène ou encore, allant à son encontre, etc. Il s'agirait donc d'identifier les capacités que l'on juge être les plus importantes et de prendre les moyens pour les développer individuellement et collectivement.

De la même manière, une analyse des façons dont les différents acteurs.trices de J'aime Hydro et de Bras de fer construisent ou minent leur propre expertise ou celle des autres pourrait être effectuée à l'aide de l'outil appelé "planche de jeu de l'expertise " proposé par Jasanoff (2008). Une telle analyse pourra être réinvestie pour examiner des discours relatifs à la crise climatique : qui présente-t-on comme expert.e.? Comment justifie-t-on ou remet-on en question son expertise? En s'appuyant sur les trois modèles identifiés par Callon (1998) et que nous avons présentés plus haut, les manières dont les relations entre citoyen.ne.s et scientifiques se déploient dans ces œuvres pourraient aussi être étudiées. Une réflexion au sujet des relations entre citoyen.ne.s et scientifiques lorsqu'il est question des changements climatiques pourrait ensuite être menée. En somme, à partir de ces œuvres, il s'agit d'examiner les capacités que des citoyen.ne.s peuvent développer et de s'approprier des outils théoriques permettant de mieux comprendre de telles situations. Ces capacités et outils pourront être mis à contribution dans la lutte aux changements climatiques ou lorsque d'autres questions technoscientifiques socialement vives émergeront. 


\section{Reconnaitre les capacités citoyennes et combattre l'idée du déficit}

En conclusion, lorsqu'il s'agit d'aborder des questions technoscientifiques environnementales et sanitaires en classe, et particulièrement lorsqu'il s'agit d'éduquer aux changements climatiques dans un contexte où l'urgence d'agir n'a plus à être discutée, un enseignement plus critique et politisé de ces questions nous apparaît incontournable. De notre point de vue, il importe de mettre de l'avant les capacités des citoyen.ne.s à s'approprier ces questions, puisque cela peut contribuer à former des personnes qui peuvent agir, à leur façon, pour la construction d'un monde plus juste et sain. En tant qu'enseignant.e.s et chercheurs.euses, nous pouvons y contribuer en reconnaissant et valorisant les capacités citoyennes, et en combattant l'idée de déficit citoyen.

\section{BIBLIOGRAPHIE}

Akrich, M., Barthe, Y., et Rémy, C. (Dir.). (2010). Sur la piste environnementale. Menaces sanitaires et mobilisations profanes. Paris : Presses des Mines.

Albe, V. (2005). Un jeu de rôle sur une controverse socio-scientifique actuelle : une stratégie pour favoriser la problématisation ? Aster, (40), 67-94.

Arseneau, I. (2019, mars). Points de vue de scientifiques agissant dans le contexte de questions environnementales ou sanitaires : quelles implications pour l'enseignement des sciences? 12e Symposium étudiant du CRIEVAT, Université Laval, Québec.

Arseneau, I., et Groleau, A. (2020). La pièce J'aime Hydro en classe de technosciences. Spectre, 49(3), 16-19.

Batellier, P., et Maillé, M.-È. (2017). Acceptabilité sociale : sans oui, c'est non. Montréal :

Écosociété.

Beaulieu, C. (2019). J’aime Hydro. Montréal : Atelier 10.

Bencze, L. (2017). Science and Technology Education Promoting Wellbeing for Individuals, Societies and Environments. (L. Bencze, Dir.). Springer.

Bencze, L., et Carter, L. (2011). Globalizing students acting for the common good. Journal of Research in Science Teaching, 48(6), 648-669.

Callon, M. (1998). Des différentes formes de démocratie technique. Annales Des Mines, (9), 63-73.

Callon, M., Lascoumes, P., et Barthe, Y. (2001). Agir dans un monde incertain. Essai sur la démocratie technique. Paris : Éditions du Seuil.

Charlot, B. (1997). Du rapport au savoir. Éléments pour une théorie. Paris : Anthropos.

Groleau, A. (2017). Rapports aux experts et aux expertes scientifiques de futures enseignantes du primaire : Construction de quatre idéaux-types. Thèse de doctorat inédite, Université Laval. 
Groleau, A. (2019). Éviter autant la dérive relativiste que la dérive autoritariste en classe de sciences et de technologie à l'ère des fausses nouvelles. Spectre, 48(3), 18-20.

Groleau, A., et Pouliot, C. (2014). Le jeu de société Decide pour s'informer, discuter et prendre position au sujet de controverses sociotechniques actuelles. Spectre (43), C2-C4.

Irwin, A. (1995). Citizen Science : A Study of People, Expertise and Sustainable Development. London : Routhledge.

Irwin, A. (2014). From deficit to democracy (re-visited). Public Understanding of Science, 23(1), 71-76.

Jasanoff, S. (2008). Representation and re-presentation in litigation science. Environmental Health Perspectives, 116(1), 123-129.

Legardez, A., et Simonneaux, L. (2006). L'école à l'épreuve de l'actualité. Enseigner les questions vives. (ESF Éditeur, Ed.). Paris.

Maingain, A., et Dufour, B. (2002). Approches didactiques de l'interdisciplinarité. (G. Fourez, Dir.). Bruxelles : De Boeck \& Larcier s.a.

Pouliot, C. (2009). Using the deficit model, public debate model and co-production of knowledge models to interpret points of view of students concerning citizens' participation in socioscientific issues. International Journal of Environmental and Science Education, 4(1), 49-73.

Pouliot, C. (2015). Quand les citoyens.ne.s soulèvent la poussière : la controverse autour de la pollution métallique à Limoilou. Montréal : Carte blanche.

Pouliot, C. (2019). Éducation aux démarches d'enquête citoyennes. Dans J. Simonneaux (Dir.), La démarche d'enquête : Une contribution à la didactique des questions socialement vives (pp. 115128). Dijon : Educagri.

Pouliot, C. et Godbout, J. (2014). Thinking outside the "knowledge deficit" box : Scientists could achieve more fulfilled professional lives by embracing the skills needed for effective interaction with the public. EMBO Reports, 15(8), 833-835.

Ripple WJ, Wolf C, Newsome TM, Barnard P, Moomaw WR. 2019. World Scientists' Warning of a Climate Emergency. BioScience, biz088.

Seaborn, J.-L., et Seaborn, J. (2017). Bras de fer. Documentaire. 77 minutes.

Sjöström, J., et Eilks, I. (2018). Reconsidering Different Visions of Scientific Literacy and Science Education Based on the Concept of Bildung. Dans Y. J. Dori, Z. Mevarech, et D. Baker (Dir.), Cognition, metacognition, and culture in STEM education (pp. 65-88). Dordrecht : Springer.

Wynne, B. (1991). Knowledges in Context. Science, Technology \& Human Values, 16(1), 111-121.

Wynne, B. (1992). Misunderstood misunderstanding : social identities and public uptake of science. Public Understanding of Science, 1, 281-304.

Wynne, B. (2006). Public engagement as a means of restoring public trust in science - Hitting the notes, but missing the music? Community Genetics, 9(3), 211-220.

\section{NOTES}

1. Alors qu'une posture non déficitaire consisterait à ne pas adopter une posture déficitaire. 


\section{RÉSUMÉS}

Dans ce texte, nous abordons la nécessité d'une éducation aux changements climatiques qui s'appuie sur les capacités citoyennes. Nous présentons d'abord le concept de déficit citoyen, puis nous discutons des raisons pour lesquelles il est important de s'intéresser aux capacités citoyennes dans les recherches en didactique des sciences et dans l'enseignement des technosciences. Cela nous mène à proposer différentes façons de mettre en lumière les capacités citoyennes et de contribuer à leur développement afin de former des personnes capables de comprendre les tenants et les aboutissants des questions environnementales et sanitaires contemporaines, de participer aux discussions sociopolitiques et d'agir sur les situations qui les concernent.

In this text, we address the need for a climate change education that is based on citizen capacities. First, we present the deficit model. Then, we discuss the reasons why it is important for research and teaching in science education to focus on citizen capacities and emphasize them. This leads us to different ways to enlighten the idea of citizen capacities, and to contribute to their development, for the training of people capable to understand the ins and outs of contemporary environmental and health issues, to participate in socio-political conversations and to act on situations they are concerned with.

\section{INDEX}

Mots-clés : déficit citoyen, posture antidéficitaire, capacités citoyennes, éducation aux changements climatiques, didactique des sciences, sociologie des sciences

Keywords : deficit model, anti-deficit posture, citizen capacities, climate change education, science education, sociology of science

\section{AUTEURS}

\section{AUDREY GROLEAU}

Audrey Groleau est professeure de didactique des sciences et de la technologie à l'Université du Québec à Trois-Rivières. Ses recherches portent sur les rapports à l'expertise scientifique de futurs enseignant.e.s, scientifiques et ingénieur.e.s dans le contexte de controverses sociotechniques actuelles. Elle contribue à la formation d'enseignant.e.s au primaire, au secondaire et en adaptation scolaire, en plus de futur.e.s chercheurs.euses en éducation. Elle détient un baccalauréat en physique et a enseigné cette discipline au collégial.

\section{ISABELLE ARSENEAU}

Isabelle Arseneau (MA, B.Ens.) est doctorante en didactique des sciences et professionnelle de recherche pour la Chaire de leadership en enseignement des sciences et développement durable à l'Université Laval, à Québec. Dans sa thèse, elle documente les points de vue de scientifiques qui s'engagent dans le contexte de questions socialement vives environnementales ou sanitaires. Ses préoccupations de recherche s'inscrivent dans une vision de l'enseignement des sciences critique, politique et orientée vers l'action citoyenne. 


\section{CHANTAL POULIOT}

Chantal Pouliot est professeure titulaire de didactique des sciences à la Faculté des sciences de l'éducation de l'Université Laval et chercheuse au Centre de recherche et d'intervention sur la réussite scolaire (CRIRES). Ses travaux de recherche concernent la documentation et l'enseignement de controverses environnementales et sanitaires actuelles. Elle a rédigé Quand les citoyen.ne.s soulèvent la poussière : la controverse autour de la poussière métallique à Limoilou (2015, Carte blanche). 